\title{
LE MAREE ALL'ISOLA DI S. ANDREA NELL'ADRIATICO CENTRALE
}

\author{
Silvio Polli
}

1. Premesse. - L'isola di S. Andrea emerge quasi al centuo del hacino mediano del mare Adriatico, $30 \mathrm{~km}$ a ovest dell'isola di Lissa.

E separata da questa da una sella subacquea profonda circa $100 \mathrm{~m}$. Y'isola ì lunga $4 \mathrm{~km}$ e larga $1,2 \mathrm{~km}$. Si cleva alquanto ripida sino ad una altezza di $305 \mathrm{~m}$ sul livello del mare. L'isohata di $100 \mathrm{~m}$ la circonda ad una distanza di $2-3 \mathrm{~km}$. Le coordinate geografiche del punto corrispondente alla massima quota sono:

$$
\begin{array}{ll}
\text { long. } & 15^{\circ} 45^{\prime} 30^{\prime \prime} \text { E. Gr. } \\
\text { lat. } & 43^{\prime \prime} 01^{\prime} 40^{\prime \prime} \mathrm{N} .
\end{array}
$$

Per la sua posizione centrale essa presenta notevole interesse nello studio delle maree e dei livelli medi del mare Adriatico. Infatti, già il generale R. von Sterneck, verso il 1900, trattando questi argomenti, vide sulito l'importanza che avrebhe un mareografo in tale isola. Nel 190t, sotto la sua direzione, fece costruire due semplici ma sicuri registratori; uno per l'isola di Pelagosa, l'altro per quella di S. Andrea. Nella prima lo strumento funzionò da fine maggio al giorno 26 agosto nel quale un violento temporale lo rese inservibile. Nella seconda fu messo nei primi giomi di ottohre del 1904 e registrò le maree regolarmente sino a tutto il mese di dicembre.

Lo Sternerk pubblico nel 1905 i dati delle letture orarie nelle: - Mitteilungen des k.u.k. Militïrgcographischen Institutes, XXIV Band ", calcolando i livelli medi mensili e confrontandoli con quelli dei porti di Trieste, Pola c Ragusa.

Esaminando tali dati orari pensai di usufruirli per calcolare le costanti armoniche e non armoniche delle maree di quella zona. Essi risultano regolari, senza interruzioni c omogenei. Ciò è confermato dai risultati delle analisi che furono appositamente esẹ̣ute indipendentemente per ciascuno dei mesi considerati.

2. Motodo di calcolo. - Per la determinazione delle costanti armoniche si adottò il metodo di analisi armonica elaborato da A. T. 
Doodson e pubblicato nclla "The Admiralty Tides Tables ", lart III, Instruction and Tables, Hydrogr. Departm., London, 1936. Dei tre procedimenti indicati si seguì quello che si applica ad una successione continua di valori orari estesa a 696 ore (29 giorni). Con i dati disponibili è possibile applicare a serie successive tale procedimento. Questo calcolo separato per ciascun periodo di 29 wiorni si priustifica coi fatti di avere un controllo della omogeneita e regolarita delle serie consi-

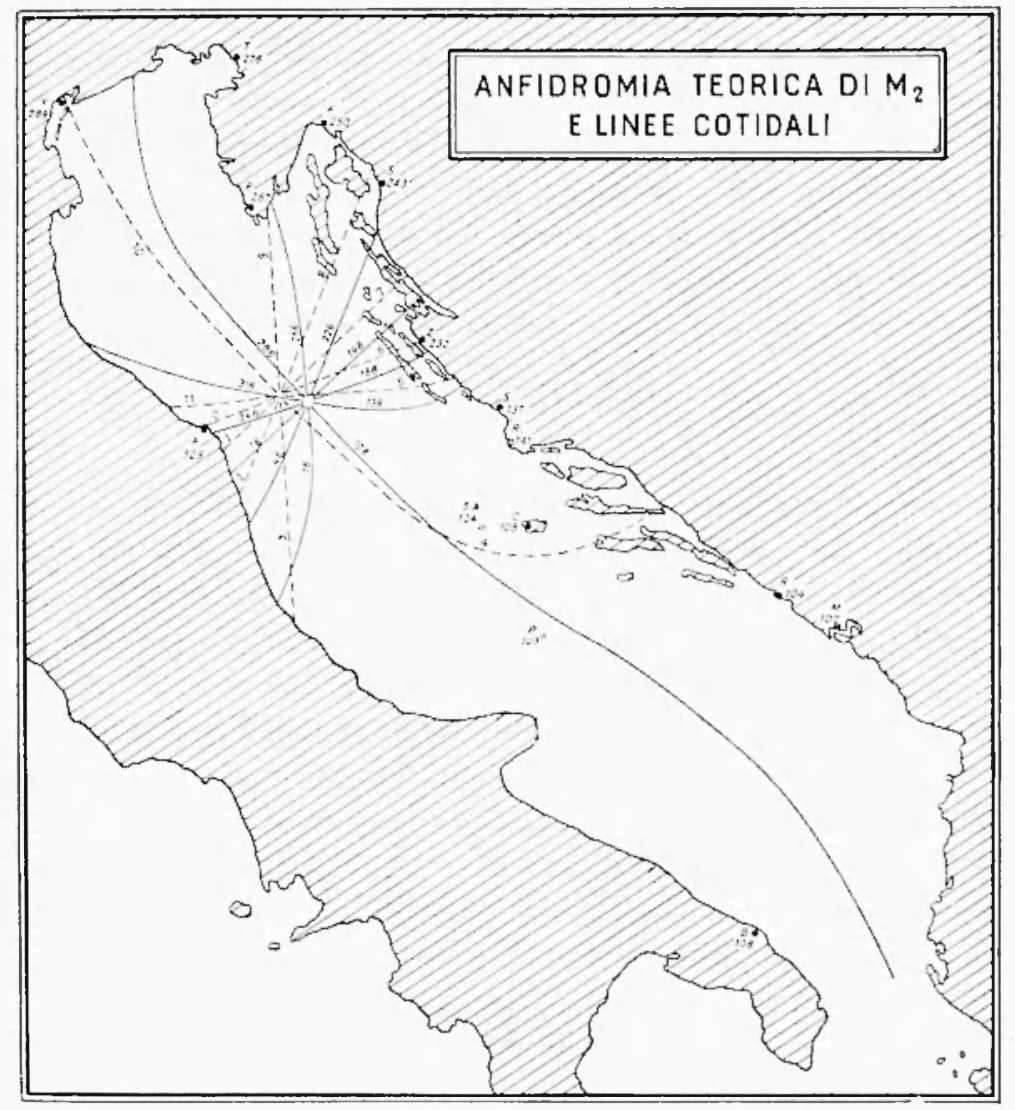

derate e di poter adoperare $\mathrm{i}$ singoli valori mensili delle costanti armoniche per altre ricerche, come quella sulla loro oscillazione stagionale in dipendenza della variazione dei vari fattori geofisici e in particolare quelli meteorici. Quali costanti armoniche di quel posto, si considerarono le medie arimetiche dei valori comi-pondenti dei 3 gruppi.

Il tempo arlottato i quello medio dell'Europa Centrale (T.M.F.C.p 
Le ampirese (ingl. amplitude, terl. Tidenhub) e le semiampiez=e II somo late: in inl.

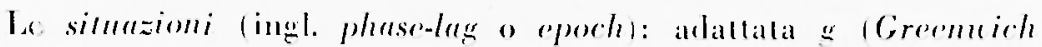
eporin e locille $\%$, sono espresse in grarli sessagresimali.

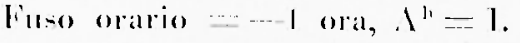

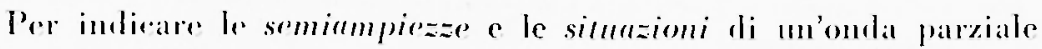
metheremo i smboli $I I, z, g$, somplicemente davanti a quelli delle onde compronenti considerate. Cosi per es. $/ K_{1}$ indichera la semianprezal dellombla parziale $K_{1}$.

Le ront. II e g per le ombe $K_{2}$ e $P_{1}$ si dedusero dalle componenti $S_{2}$. K. moliante i rapporti

$$
\begin{aligned}
& 11 K_{2}=0.2711 \mathrm{~S}_{2} \quad g K_{2}=g ._{\mathrm{n}} \\
& H H_{1}=0.33 \mathrm{HK} K_{1} \quad g I_{1}=g \Lambda_{1}
\end{aligned}
$$

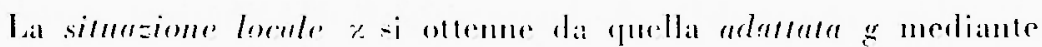
la relazions:

$$
\left.x=g+(p) i^{0}-n^{0 \prime} \Lambda^{\mathrm{h}}\right)
$$

dove: $p=$ mationo dell'simlat.

$\because^{\prime \prime}=$ longiturlime da Gerenwirh.

$u^{\prime \prime}=$ meleciti oraria dellouma.

$l^{\prime \prime}=$ loigutudine in ore del meridiano sul quale i resolato lorulonio.

3. Meduzione delie costunli non! armoniche. -.- Dalle costanti armoniche si calcolarono funlle non armoniche modiante lo seguenti formule:

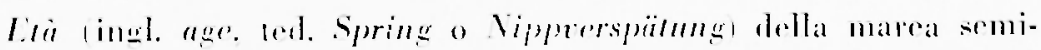
limma in piorni:

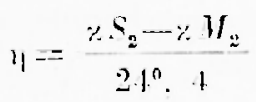

Stabitimente medio del porto a intervallo medio delle alte maree tingl. mean high water inteital, corrected establishment of the portl:

$$
\mid H A 111111)=\frac{* 1 \%}{29}
$$

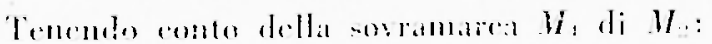

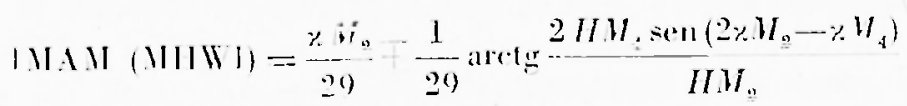


love il segno - vale prer le alte maree e quello th per le basse.

Stabilimento edgare del porto o intervallo siriqiale della alta matra fingl. high water full and change, common or vulgar establishmont of the portl:

$$
\text { ISAM (IIWFN })=\frac{{ }_{1} H_{2}}{29}-\frac{1}{30} \operatorname{arctg} \frac{\operatorname{sen}\left(\varkappa M_{2}-x S_{2}\right)}{\frac{H H_{2}}{H S_{2}}+\cos \left(x H_{2}-x S_{4}\right)}
$$

Alteza dol livello medio o dislivello tra il piano di riduzione regli scamblagli e il livello medie del mare o semplicemente livello

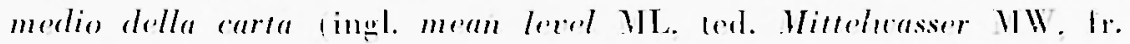
nivenu moyen $\mathrm{X} / 1$.

$\mathrm{MI},=52$ ('m1.

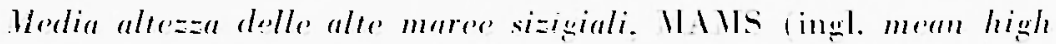
water me'ap. MH $\mathrm{NI}$ :

I) contata dal livello medio: HW. 11 S..:

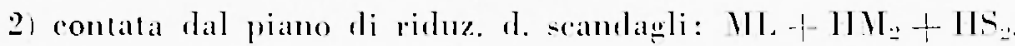

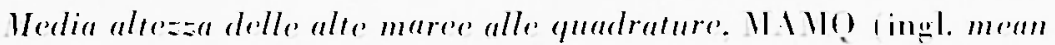
high water sprins. MIIISS:

II comtatat dal livello medio: HII, HS:

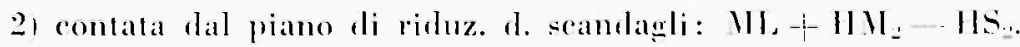

Massimo imalzanento possibile dellalea marea e depresione mas-ima della hassa marea rispetlo al livelle medio del mare. considerati quali somme delle alteze delle nowe componenti:

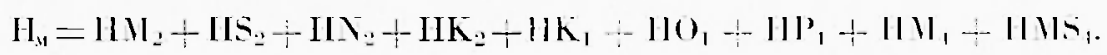

1. Le rostemti armonirhe. -. Furomo analizati i sewnenti periodi di 696 ore dellanno 1901: dalle ore 0 del wiono 6 ottobre alle ore 23

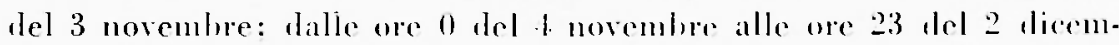
bre: dalle ore 0 del 3 dicembre alle ore 23 del 31 dicemhere. Le rostanti armoniele corrispondenti si presentano nella seguente labella.

5. Le costanti non armoniche. - Età dolla marea semidiurna: $1=0.11$ ariorni.

Stabilimento del porto o intervallo merlio delle alte mares:

IMIII (MHWI $=4.28$ ore $=f^{\mathrm{n}} 17^{\mathrm{m}}$,

tenendo conto della sovaunarea $H_{4}$ di $W_{\text {., }}$ : 
Le costanti armoniche allisola di S. Andrea

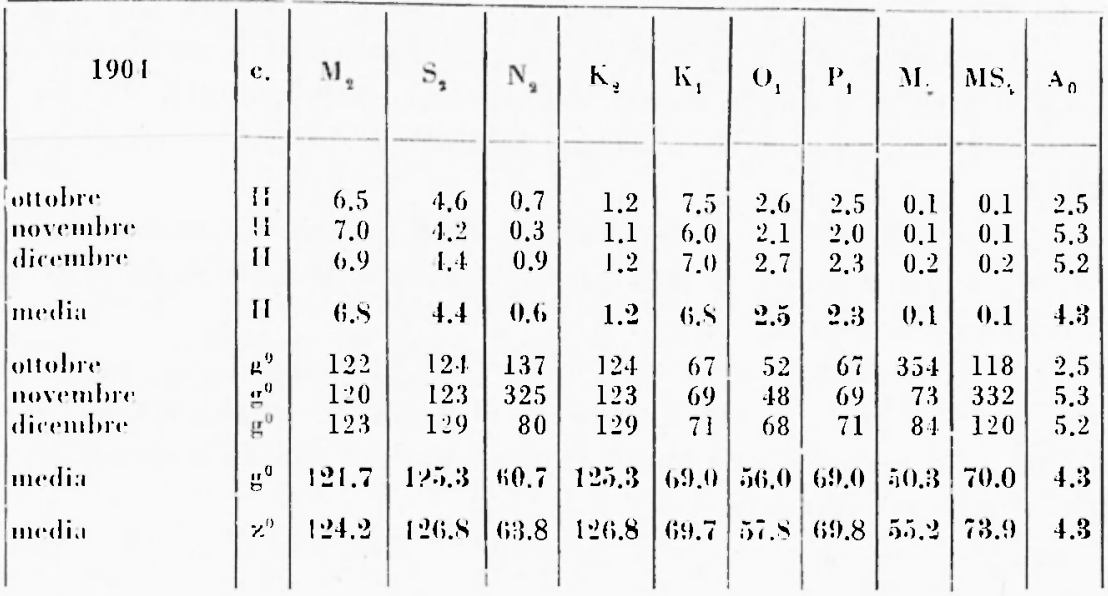

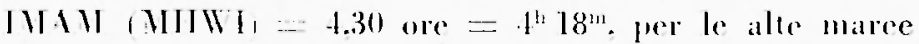
$1111111111 W 1=1.27$ orr $=1^{\mathrm{th}} 16^{\mathrm{m}}$. per le hasse maree.

Stabilimento roluare del porto o intervallo sizigiale dellalta marea:

ISAII , IIIIFEC = 1.32 ore $=.^{\mathrm{th}} 19^{\mathrm{mu}}$.

Dislivello tra il piano di riduzione degli scambạli e il l.m.m.:

III $=52 \cdot \mathrm{ml}$.

Hlez=a media delle alte maree sizisiali:

contala dal livello medio: 11.2 rm:

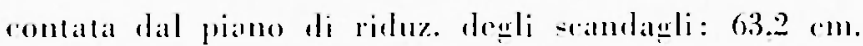

Hlezan modia delle aler maree alle quadrature:

contata dal livello medio: 2.t coll:

rontata dal piano di riduz. deg̣li scamdanli: $5-4.4$ rm.

lessimo immalsamento possibile eomma delle nove $H$ : $24.8 \mathrm{~cm}$.

6. Considerasioni o confromti. -.- Ia marec sono di tipo prevalentemente somidiumo. In vicinanza alle quadrature diventano diurne. Risulta pertanto soddistalta la disuguaglianzal $M M_{2}-H S_{2}<M K_{1}+$

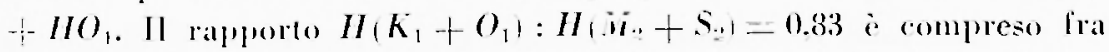
i valori 0.25 a 1.25: la marea is a lipe mi-to secomblo la nomenclatura del Van der Stok. Questo valure is pure compreso lia quelli medi 
corrispondenti all helriatieo settentrionale o entro-merielionale elue $=01100.93$ i. 0.61 .

II ripporto $I I S:$ : $H U_{2}=0.65$ i superiore tanto a quello treorico $(0,-465)$ quanto a quello medio dell' Adriatien settentrionals 10.561 , $\therefore$

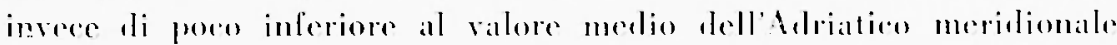
(0.63).

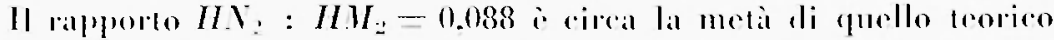
(0,19), al quale invere le stazioni dell'Ariatico settentrionale si avirinamo noterolmente.

Alto rispetto al valore teorien 10.100 risalta il rapperto

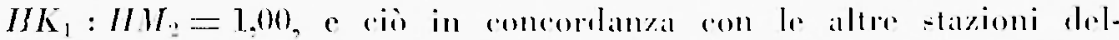

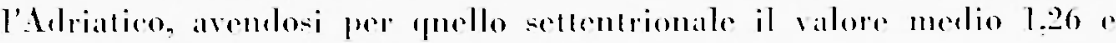
per quello merielionale 0.73.

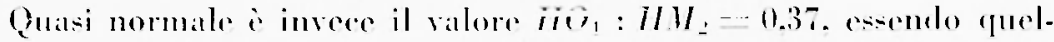
lo teorico 0.12 .

L'ampiezar dell'onta lunisolane derlinarionale dimrna $K_{1}=\mathbf{6 . 8 3}$

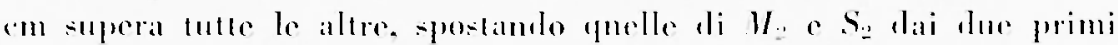
posti. Questo arviene pure per Ineonil. Pesare a per tulte le starioni

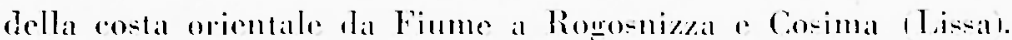

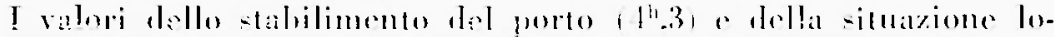

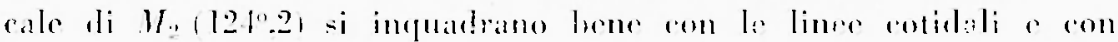
l'anfidromia teoriea di $h_{22}$ determinata dallo Sterneek. Anzi spostan-

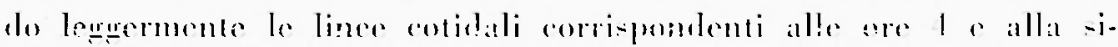

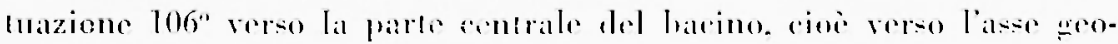

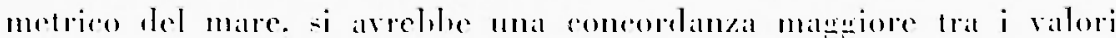

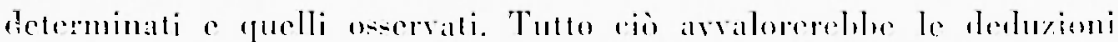
teoriche dello stemesk.

Anche l'andiamento delle alterae dei livelli medi mensili. ahe risultano pure alallanalisi stesas. i in huon acromlo tanto con furello

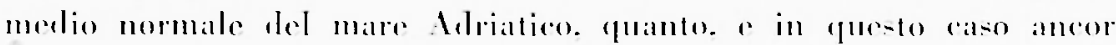
meglio, con quello del mare Tonio.

Trieste - Istiluto Talassografico -. Sollombro 1950.

\section{RIASSUTO}

Si calcolano le costanti armoniche " non armoniche delle maroes allisola di S. Andrea nell Adriation centrale. Si segue il procedimento slaborato da T. Doodson applicandolo per an periodo di tre mesi. 
le maree sono di lipo prevalentemente semidiurno, in vicinanza alle" quadrature diventano diurne. I valori delerminali sono in buon accordo con l'anfidromia teorica calcolata dallo Sterneck.

\section{IBIIIIOOFR:IIIA}

Donosu、 A. T. - Wamb ni; 1I. D., The Admirnl/y Tides Tnbles. Part III, Instruc.

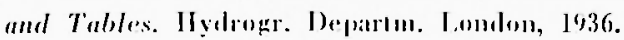

Polla S.: Oscillazione anmu dol Mare Mediuerraneo. Arch, di Oceanogr. e limn. Vol. 1. 1. Mem. 298 Comil. 'Talass. 11., 1011.

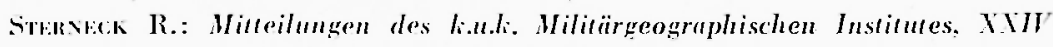
Band, 190.5, Wien.

Strmseck R. D.: Die Cezeilenerscheinungen in der Adria, II. Teil. Denkschr. M. Wi-.. Wirn. MAT.-NAT. KI.. B. 96, 1. Wien, 1919.

Traxi M.: Haree e correnti di marea. Ist, Idrog. della Marina, 1935, 1910.

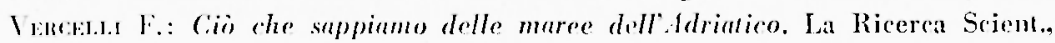
Roma, 9-11. 1932. 